\title{
Environmental risk factors and comorbidities of primary biliary cholangitis in Korea: a case-control study
}

\author{
Kyung-Ah Kim ${ }^{1}$, Young Seok Kim², Sang Hoon Park ${ }^{3}$, Woo Jin Chung ${ }^{4}$, Dae Hee Choi ${ }^{5}$, Eun Sun Jang ${ }^{6}$, \\ and Sook-Hyang Jeong ${ }^{6}$
}

\begin{abstract}
${ }^{1}$ Department of Internal Medicine, Inje University Ilsan Paik Hospital, Goyang; ${ }^{2}$ Department of Internal Medicine, Soonchunhyang University Bucheon Hospital, Bucheon; ${ }^{3}$ Division of Gastroenterology and Hepatology, Hallym University Kangnam Sacred Heart Hospital, Seoul; ${ }^{4}$ Department of Internal Medicine, Keimyung University School of Medicine, Daegu; ${ }^{5}$ Department of Internal Medicine, Kangwon National University School of Medicine, Chuncheon; ${ }^{6}$ Department of Internal Medicine, Seoul National University Bundang Hospital, Seongnam, Korea
\end{abstract}

Received: July 19, 2019

Revised : August 28, 2019

Accepted: September 5, 2019

\section{Correspondence to}

Sook-Hyang Jeong, M.D.

Department of Internal Medicine, Seoul National University Bundang Hospital, 82 Gumi-ro 173 Beon-gil, Bundang-gu, Seongnam 13620 , Korea

Tel: +82-31-787-7034

Fax: $+82-31-787-4052$

E-mail:jsh@snubh.org

https://orcid.org/0000-00024916-7990
Background/Aims: The risk factors for the development of primary biliary cholangitis ( $\mathrm{PBC})$ is unclear. This study aimed to investigate the risk factors associated with $\mathrm{PBC}$ in Korea through a questionnaire survey.

Methods: Consecutively enrolled 103 PBC patients from six referral hospitals and 100 age- and sex-matched community controls participated in this study. A standardized questionnaire survey including demographics, lifestyle, individual and familial medical history and reproductive history was prospectively collected and analyzed.

Results: The PBC patients had a mean age of 58.3 years and a female proportion of $86.4 \%$. The age- and sex-matched controls had a similar educational level and economic status to the PBC patients. Among the lifestyle factors, the multivariable analysis showed smoking including both first-hand and second-hand (odds ratio [OR], 2.03; 95\% confidence interval [CI], 1.06 to 3.93), history of autoimmune diseases (OR, 2.46; 95\% CI, 1.06 to 6.35), and family history of PBC (OR, 17.76; 95\% CI, 1.77 to 2,418.74) were significantly associated with PBC, whereas alcohol intake was negatively associated with $\mathrm{PBC}$. Among reproductive factors, the number of induced abortions was significantly associated with $\mathrm{PBC}$, while the number of full-term deliveries was negatively associated with PBC.

Conclusions: A family history of $\mathrm{PBC}$, accompanying autoimmune diseases, and smoking were significantly associated with PBC. More induced abortions and less full-term deliveries were associated with PBC in women. In contrast, mild to moderate alcohol intake was negatively associated with PBC. Further studies are warranted to validate the results of this study and to search for clues about the pathogenesis of $\mathrm{PBC}$.

Keywords: Liver cirrhosis, biliary; Risk factors; Smoking; Ethanol; Abortion, induced

\section{INTRODUCTION}

Primary biliary cholangitis (PBC) is a rare, chronic inflammatory autoimmune disease that mainly affects middle-aged women [1]. It is characterized by non-suppurative inflammation and destruction of the interlobular bile ducts leading to cholestasis and cirrhosis and the presence of the anti-mitochondrial antibody (AMA) 
in serum [1].

The prevalence of $\mathrm{PBC}$ varies widely depending on the geographic location, ranging from 1.91 to $40.2 \mathrm{per}$ 100,000 inhabitants [2], which suggests certain genetic and environmental factors influence the pathogenesis of PBC. Though the pathogenesis of PBC still remains enigmatic, an organ-specific autoimmune reaction triggered by exposure to putative environmental factors in genetically susceptible individuals is a convincing explanation [3].

Previous studies regarding the environmental risk factors of PBC have been conducted mostly in Western countries with a relatively high prevalence of PBC. Those studies reported that smoking [4-7] and recurrent urinary tract infection (UTI) were frequently associated with PBC. However, the extent of exposure to environmental factors such as smoking, alcohol drinking, use of cosmetics, or contraceptive measures is variable depending on the countries and regions with different epidemiologies and life styles. In this case-control study, we investigated the environmental risk factors and comorbidities associated with PBC in South Korea.

\section{METHODS}

\section{Study population}

One hundred-three patients with PBC from six referral centers in South Korea were prospectively enrolled from August 2014 to March 2015. The diagnostic criteria for $\mathrm{PBC}$ were at least two of the following findings present in the patients; chronic cholestasis, presence of AMA and liver histology compatible with PBC. The Institutional Review Board (IRB) approved this study (Ilsan Paik Hospital IRB number 2014-08-233) and written informed consent was received from all participants. The patients diagnosed as autoimmune hepatitis-PBC overlap syndrome were excluded.

As a control group, 100 individuals matched to the PBC cases according to a stratified sampling based on age (10-year age groups) and gender were recruited from the commercialized Macromill Embrain research panel (Macromill Embrain, Seoul, Korea; www.embrain.com), which consists of 1,088,408 individuals representative of the Korean national population. The participants were enrolled on a voluntary basis. The survey was conducted anonymously between August 25 and September 26, 2014.

\section{Questionnaire survey}

A standardized questionnaire survey was administered that included 137 questions regarding demographic, anthropometric, life style, medical and familial factors, reproductive (only for female) and socioeconomic factors. Demographics included education and subjective socioeconomic class. Lifestyle factors included first- and second-hand smoke, alcohol consumption (drinks per day, frequency and duration), coffee consumption, and use of cosmetics and hair dye. Medical history included autoimmune diseases such as autoimmune hepatitis, thyroid illness, rheumatoid arthritis, systemic lupus erythematosus, systemic sclerosis, Sjogren's disease, and inflammatory bowel diseases, infectious disease such as tuberculosis, clonorchiasis and measles, operations and vaccinations. Reproductive factors included age of menarche and menopause, oral contraceptives, hormone replacement therapy, induced abortion, miscarriage, full-term delivery, and breast feeding. The details of the questionnaire (in Korean) is presented in the Supplementary Material.

PBC patients were interviewed face-to-face by a research coordinator or attending physician at each of the centers. Controls were interviewed face-to-face by trained persons hired by Macromill Embrain.

\section{Statistical analysis}

Continuous variables were expressed as the median (interquartile range) or mean $\pm \mathrm{SD}$ as appropriate. Categorical variables are presented in absolute and percentage values (\%). Continuous variables were compared using the Student's $t$ test, or Mann-Whitney $U$ test as appropriate, and categorical variables were compared using the chi-square test or Fisher's exact test. Multivariable logistic regression analyses with the Firth method for rare events were performed for variables with a $p<0.1$ in the univariable analysis and for clinically relevant variables with $p>0.1$, but which were consistently reported as significant variables in other studies (i.e., smoking and UTI) to identify the risk factors of PBC for all subjects and a female subgroup, respectively. A $p<0.05$ was considered statistically significant. All statistical analyses were performed with SPSS version 25 (IBM Co., Armonk, NY, USA). 
Table 1. Demographic and socioeconomic characteristics of PBC cases and controls

\begin{tabular}{lccc}
\hline Characteristic & $\begin{array}{c}\text { PBC cases } \\
(\mathrm{n}=103)\end{array}$ & $\begin{array}{c}\text { Controls } \\
(\mathrm{n}=100)\end{array}$ & p value \\
\hline Female sex & $86.4(90)$ & $85(85)$ & 0.77 \\
\hline Age at enrollment, yr & $58.6 \pm 11.4$ & $56.8 \pm 10.8$ & 0.26 \\
\hline Age at diagnosis, yr & $55.2 \pm 10.4$ & - & \\
BMI, kg/m ${ }^{2}$ & $23.2 \pm 2.6$ & $23.0 \pm 2.3$ & 0.51 \\
Educational level & & & 0.25 \\
$\quad$ Middle school & $23.5(24)$ & $25(25.3)$ & 0.50 \\
$\quad$ High school & $42.2(43)$ & $50(50.5)$ & \\
$\geq$ College & $34.3(35)$ & $24(24.2)$ &
\end{tabular}

Economic status

\begin{tabular}{ccc} 
High & 0 & $2.1(2)$ \\
Mid & $71.8(28)$ & $76.3(74)$ \\
Low & $28.2(11)$ & $21.7(21)$ \\
\hline
\end{tabular}

Values are presented as \% (number) or mean \pm SD.

$\mathrm{PBC}$, primary biliary cholangitis; BMI, body mass index.

\section{RESULTS}

\section{Comparison of the demographic, socioeconomic, lifestyle factors, and medical history between the PBC patients and control group}

The mean age of the case and control group was $58.6 \pm$ 11.4 and $56.8 \pm 10.8$ years, respectively. The female proportion of the case and control group was $86.4 \%$ and $85 \%$, respectively. Educational level and economic status were similar between the two groups (Table 1 ).

The proportions of ever-smokers including current and ex-smoker (15.5\% vs. $11.0 \%)$ were low in both groups and not statistically different between the PBC and controls groups (Table 2). Although the proportions of those who have experienced first-hand and/or second-hand smoking tended to be higher in the PBC patients (49.5\%) than in the control (42.0\%) group, it was not significantly different $(p=0.16)$. The proportions of ever-drinkers and persons who have had more than seven drinks per

Table 2. Lifestyle factors of PBC cases and controls

\begin{tabular}{|c|c|c|c|}
\hline Variable & PBC cases $(n=103)$ & Controls $(n=100)$ & $p$ value \\
\hline \multicolumn{4}{|l|}{ First-hand smoking } \\
\hline Ever regularly smoked & $16 / 103(15 \cdot 5)$ & $11 / 100(11.0)$ & 0.41 \\
\hline Current smoking & $5 / 103(4.8)$ & $7 / 100(7.0)$ & 0.56 \\
\hline Second-hand smoke exposure & $42 / 99(42.4)$ & $37 / 99(37.4)$ & 0.56 \\
\hline Smoking including both first- \& second-hand & $51 / 98(49 \cdot 5)$ & $42 / 100(42.0)$ & 0.16 \\
\hline Female & $39 / 84(42.6)$ & $33 / 85(38.8)$ & 0.32 \\
\hline Male & $12 / 14(72.4)$ & $9 / 15(60.0)$ & 0.12 \\
\hline Alcohol drinking, $\mathrm{A} / \mathrm{B} / \mathrm{C}^{\mathrm{a}}$ & $9 / 32 / 62(8.7 / 30.1 / 61.2)$ & $13 / 53 / 34(13.0 / 53.0 / 34.0)$ & $<0.01$ \\
\hline Female & $5 / 24 / 60(5.6 / 27.0 / 67.4)$ & $6 / 45 / 34(7.1 / 52.9 / 40.0)$ & \\
\hline Male & $4 / 8 / 2(28.6 / 57 \cdot 1 / 14 \cdot 3)$ & $7 / 8 / 0(46.7 / 53.3 / 0)$ & \\
\hline Coffee & $89 / 103(86.4)$ & $92 / 100(92.0)$ & 0.20 \\
\hline Hair dye (>2/yr) & $33 / 101(32.7)$ & $30 / 99(30.3)$ & 0.72 \\
\hline Female & $32 / 87(36.8)$ & $27 / 84(32.1)$ & \\
\hline Male & $1 / 14(7.1)$ & $3 / 15(20.0)$ & \\
\hline Nail polish (> 2/yr) & $13 / 102(12.7)$ & $18 / 100(18.0)$ & 0.30 \\
\hline Female & $13 / 88(14.8)$ & $17 / 85(20.0)$ & \\
\hline Male & $0 / 14(0.0)$ & $1 / 15(6.7)$ & \\
\hline Hair perm (> 4/yr) & $36 / 102(35 \cdot 3)$ & $34 / 100(34.0)$ & 0.85 \\
\hline Female & $36 / 88(40.9)$ & $33 / 85(38.8)$ & \\
\hline Male & $0 / 14(0.0)$ & $1 / 15(6.7)$ & \\
\hline
\end{tabular}

Values are presented as number (\%).

PBC, primary biliary cholangitis.

${ }^{\mathrm{a}} \mathrm{A}, \geq 7$ drinks/week for female, $\geq 14$ drinks/week for male; $\mathrm{B},<7$ drinks/week for female, $<14$ drinks/week for male; C, never drinker. 
Table 3. History on the accompanying disease and family history in PBC cases and controls

\begin{tabular}{|c|c|c|c|}
\hline Variable & PBC cases $(n=103)$ & Controls $(n=100)$ & $p$ value \\
\hline Sjogren's disease & $27 / 103(6.3)$ & $1 / 97(1.0)$ & 0.06 \\
\hline Raynaud phenomena & $2 / 100(2.0)$ & $0 / 96(0.0)$ & 0.50 \\
\hline Thyroid disease & $19 / 103(18.4)$ & $7 / 99(7.1)$ & 0.02 \\
\hline Any type of autoimmune disease & $27 / 103(26.2)$ & $9 / 90(9.1)$ & $<0.01$ \\
\hline Diabetes mellitus & $16 / 103(15 \cdot 5)$ & $7 / 97(7.2)$ & 0.07 \\
\hline Osteoporosis & $23 / 103(22.1)$ & $10 / 100(10.0)$ & $<0.05$ \\
\hline Urinary tract infection & $27 / 101(26.7)$ & $19 / 100(19.0)$ & 0.19 \\
\hline Acute hepatitis & $4 / 102(3.9)$ & $0 / 98(0.0)$ & 0.12 \\
\hline History of herbal medication & $80 / 103(77.7)$ & $77 / 99(77.8)$ & 0.99 \\
\hline PBC in first degree relatives & $6 / 97(6.2)$ & $0 / 97(0.0)$ & 0.02 \\
\hline Thyroid disease in first degree relatives & $9 / 103(8.7)$ & $8 / 100(8)$ & 0.85 \\
\hline Autoimmune hepatitis in first degree relatives & $1 / 103(1.0)$ & $0 / 100(0.0)$ & 1.00 \\
\hline Sjogren's disease in first degree relatives & $0 / 103(0)$ & $0 / 100(0)$ & - \\
\hline
\end{tabular}

Values are presented as number (\%).

PBC, primary biliary cholangitis.

week were significantly lower in the PBC groups than in the controls $(p<0.01)$. Coffee drinking and exposure to nail polish, cosmetics and hair dye were not significantly different between the two groups. The prevalence of $\mathrm{PBC}$ in first degree relatives was higher in the PBC cases $(6.2 \%$ vs. $\circ \%, p=0.02)$. The prevalence of other autoimmune diseases in first degree relatives did not differ between the relatives of the PBCs cases and those of the control group (Table 3).

History of any autoimmune diseases including autoimmune thyroiditis, Sjogren's disease, autoimmune hepatitis, systemic sclerosis and others was more frequent in the PBC cases than in the control group $26.2 \%$ vs. $9.1 \%, p<0.01$ ). Among the autoimmune diseases, autoimmune thyroiditis (18.5\% vs. $7.1 \%$ ) and Sjogren's disease $(6.3 \%$ vs. $1.0 \%)$ were significantly more prevalent in the PBC cases. Diabetes seemed to be more frequent in the $\mathrm{PBC}$ cases than in the controls (15.5\% vs. $7.2 \%, p=0.07)$. Medication for osteoporosis was more frequent in the PBC cases than in the controls. However, history of UTI as a possible risk factor of PBC in previous studies did not differ between the two groups. History of herbal medication was similarly frequent in both groups (78.6\% vs. $77.8 \%$ ) (Table 3 ). The frequencies for a positive history of all types of malignancies, acute hepatitis, tuberculosis, clonorchiasis, appendectomy, cholecystectomy, tonsillectomy, and vaccination for hepatitis and tuberculosis were not different between the two groups.

\section{Comparison of the reproductive and gynecological factors between the PBC patients and the control group among women}

Age at menarche and menopause was similar between both groups. The PBC patients (74.2\%) had a similar frequency in menopause state as the control group (62.5\%). Experience with both pregnancy (91\% vs. 100\%, $p<0.05$ ) and full-term delivery (91\% vs. 100\%, $p<0.05$ ) was less frequent in the $\mathrm{PBC}$ cases than in the controls. Moreover, the total number of full-term deliveries was lower in the PBC cases than in the controls $(2.1 \pm 1.2 \mathrm{vs}$. $2.5 \pm 0.9, p<0.05)$. Experience of receiving an induced abortion tended to be higher in the PBC groups than in the controls ( $51.1 \%$ vs. $37.7 \%, p=0.07$ ), and number of abortions was significantly higher in the PBC groups $(1.2 \pm 2.1$ vs. $0.5 \pm 0.7, p<0.05)$. However, spontaneous abortions were not significantly different between the two groups. The frequencies of hormonal replacement therapy for postmenopausal syndrome and past use of oral contraceptives were similar between the two groups (Table 4). Pruritus and abnormal liver biochemistry during pregnancy were not different between the two groups, either. 
Table 4. Reproductive and gynecological factors of female PBC cases and controls

\begin{tabular}{|c|c|c|c|}
\hline Variable & PBC cases $(n=89)$ & Controls $(\mathrm{n}=85)$ & $p$ value \\
\hline Age, yr & $58.4 \pm 11.1$ & $56.9 \pm 10.9$ & 0.35 \\
\hline Age of menarche, yr & $15.1 \pm 1.9$ & $14.7 \pm 1.6$ & 0.14 \\
\hline Menopaused state & $74.2(66 / 89)$ & $62.5(53 / 85)$ & 0.09 \\
\hline Age at menopause, yr & $49.8 \pm 5.0$ & $50.9 \pm 2.1$ & 0.32 \\
\hline Hormone replacement therapy & $16.7(13 / 66)$ & $7.8(4 / 53)$ & 0.18 \\
\hline \multicolumn{4}{|l|}{ Pregnancy } \\
\hline Ever & $91(81 / 89)$ & $100(85 / 85)$ & $<0.05$ \\
\hline No. of pregnancy & $3.8 \pm 0.3$ & $3.2 \pm 1.2$ & 0.17 \\
\hline Full-term delivery, ever & $91(81 / 89)$ & $100(85 / 85)$ & $<0.05$ \\
\hline No. of full-term delivery & $2.1 \pm 1.2$ & $2.5 \pm 0.9$ & $<0.05$ \\
\hline Miscarriage & $25.8(23 / 89)$ & $23 \cdot 5(20 / 85)$ & 0.72 \\
\hline \multicolumn{4}{|l|}{ Artificial abortion } \\
\hline Ever & $51.1(45 / 88)$ & $37.7(32 / 85)$ & 0.07 \\
\hline No. of abortion & $1.2 \pm 2.1$ & $0.5 \pm 0.7$ & $<0.05$ \\
\hline Use of oral contraceptives & $25 \cdot 3(22 / 87)$ & $25 \cdot 9(22 / 85)$ & 0.93 \\
\hline Pruritus during pregnancy & $4.8(4 / 84)$ & $8.3(7 / 84)$ & 0.53 \\
\hline
\end{tabular}

Values are presented as mean $\pm \mathrm{SD}$ or $\%$ (number).

$\mathrm{PBC}$, primary biliary cholangitis.

Table 5. Multivariable analysis on the risk factors for PBC in PBC cases and controls

\begin{tabular}{|c|c|c|c|c|}
\hline \multirow{2}{*}{ Factor } & \multicolumn{2}{|c|}{ Univariable analysis } & \multicolumn{2}{|c|}{ Multivariable analysis } \\
\hline & OR & $95 \% \mathrm{CI}$ & OR & $95 \% \mathrm{CI}$ \\
\hline Smoking (first- \& second-hand) & 1.50 & $0.86-2.63$ & 2.03 & $1.06-3.98$ \\
\hline \multicolumn{5}{|l|}{ Alcohol $^{\mathrm{a}}$} \\
\hline A vs. C & 0.38 & $0.15-0.98$ & 0.31 & $0.10-0.89$ \\
\hline B vs. C & 0.33 & $0.18-0.61$ & 0.31 & $0.15-0.61$ \\
\hline Autoimmune disease, any type & 3.55 & $1.57-8.02$ & 2.46 & $1.06-6.35$ \\
\hline Family history of $\mathrm{PBC}$ in first degree relatives & 13.85 & $1.60-1,815 \cdot 30$ & $17 \cdot 76$ & $1.77-2,418.74$ \\
\hline Diabetes & 2.37 & $0.93-6.03$ & 2.64 & $0.99-7.49$ \\
\hline Urinary tract infection & 1.56 & $0.80-3.03$ & 1.52 & $0.73-3.23$ \\
\hline
\end{tabular}

PBC, primary biliary cholangitis; OR, odds ratio; CI, confidence interval.

${ }^{\mathrm{a}} \mathrm{A}, \geq 7 \mathrm{drinks/week} \mathrm{for} \mathrm{female,} \geq 14$ drinks/week for male; $\mathrm{B},<7$ drinks/week for female, < 14 drinks/week for male; C, never drinker.

\section{Multivariable analysis of the risk factors for PBC}

Results of the univariable and multivariable analyses among the whole subjects are shown in Table 5. In multivariable analysis, family history of $\mathrm{PBC}$, history of other autoimmune diseases, and smoking including first- and second-hand were significantly associated with $\mathrm{PBC}$, but alcohol drinking was negatively associated with PBC.
As a subgroup analysis including only women, the multivariable analysis showed that the presence of family history of PBC, history of other autoimmune diseases, and induced abortions were significantly associated with PBC, but alcohol dinking less than seven drinks per week and number of full-term deliveries were negatively associated with PBC (Table 6). 
Table 6. Multivariable analysis in female PBC cases and controls

\begin{tabular}{|c|c|c|c|c|}
\hline \multirow{2}{*}{ Factor } & \multicolumn{2}{|c|}{ Univariable analysis } & \multicolumn{2}{|c|}{ Multivariable analysis } \\
\hline & OR & $95 \% \mathrm{CI}$ & OR & $95 \% \mathrm{CI}$ \\
\hline Smoking (first- \& second-hand) & 1.37 & $0.74-2.52$ & 1.47 & $0.68-3.24$ \\
\hline \multicolumn{5}{|l|}{ Alcohol } \\
\hline$>7$ drinks/week vs. never & 0.47 & $0.13-1.66$ & 0.32 & $0.06-1.38$ \\
\hline Up to 7 drinks/week vs. never & 0.30 & $0.16-0.58$ & 0.16 & $0.06-0.36$ \\
\hline Autoimmune disease, any type & 3.63 & $1.59-8.29$ & 2.82 & $1.07-7.92$ \\
\hline Family history of $\mathrm{PBC}$ in first degree relatives & 13.67 & $1.57-1793.64$ & 13.28 & $1.37-1798.00$ \\
\hline No. of full-term delivery & 0.72 & $0.53-0.96$ & 0.69 & $0.48-0.97$ \\
\hline No. of abortion & 1.59 & $1.14-2.20$ & 2.22 & $1.44-3.69$ \\
\hline
\end{tabular}

PBC, primary biliary cholangitis; OR, odds ratio; CI, confidence interval.

\section{DISCUSSION}

In this age-and sex-matched case-control study using a face-to-face questionnaire interview, family history of PBC, history of autoimmune diseases, smoking including both first-hand and second-hand, and induced abortions in women were significant risk factors, but alcohol drinking and full-term delivery in women were protective for $\mathrm{PBC}$ in South Korea, where the prevalence of $\mathrm{PBC}$ is low as 4.75 per 100,000 inhabitants [8].

The pathogenesis of PBC is still unknown but is thought to be triggered by certain environmental factors which drive the loss of tolerance to mitochondrial antigens of small bile ducts in genetically susceptible hosts. The role of genetic factors in the pathogenesis of PBC is suggested by monozygotic twin concordance [9], a higher prevalence of PBC and other autoimmune diseases in first degree relatives [10], and genetic studies on major histocompatibility [11]. Environmental factors in the pathogenesis of PBC has been investigated mainly by case-control studies using questionnaire surveys on demographics, life style, medical history, and family history. Among these factors, smoking was consistently associated with PBC [4-7] or advanced fibrosis in PBC [12]. Most studies have shown that the history of recurrent UTI was positively associated with $\mathrm{PBC}$ [4-7], while alcohol intake was negatively associated with $\mathrm{PBC}$ $[6,7]$. Some studies have reported with controversy that abortions [5], exogenous estrogens [7], and frequent use of nail polish [7] or hair dye [6] were associated with an increased risk of the disease.
In our study, the first-hand smoking rate was not significantly associated with PBC in this study. However, smoking including both first-hand and second-hand was significantly associated with PBC in the multivariable analysis, although this association was abolished in the multivariable analysis only for women that included gynecological and reproductive factors. The smoking rates in our study are much lower (15.5\%) than those in the previous studies ( $45 \%$ to $53 \%$ ) [4,5] in which smoking was a significant risk factor of PBC. Moreover, the smoking rate in Korean women is much lower than in women in other countries with a high prevalence of PBC [13]. Several studies reported that cigarette smoking was not only a risk factor of $\mathrm{PBC}[4,6,7,14,15]$, but also a factor for advanced fibrosis in $\mathrm{PBC}$ in a dose-dependent manner [13], while only a few studies found no association between smoking and PBC [10,16]. Smoking is generally a profibrotic factor for advanced fibrosis in non-alcoholic fatty liver disease as well as alcoholic and viral liver disease, probably due to free radical generation, oxidative stress or hypoxia [17]. However, some discrepancies in the association of smoking and PBC might be due to the study design or sample size. Smoking is considered a contributing factor to $\mathrm{PBC}$ pathogenesis.

This study showed that alcohol drinking was negatively associated with $\mathrm{PBC}$, which was compatible with previous studies $[6,7]$. The negative association between $\mathrm{PBC}$ and alcohol intake might result from recall bias or a reduction in alcohol intake after a patient has been diagnosed with a liver disease. However, considering that the proportion of never-drinkers was much higher in 
the PBC cases, alcohol drinking was not likely to merely be a confounding factor. Moreover, the protective effects of low to moderate alcohol drinking was suggested in systemic lupus erythematosus, rheumatoid arthritis and autoimmune hepatitis $[18,19]$. Therefore, the association of alcohol and PBC needs to be explored in further studies.

In keeping with previous studies, PBC was frequently associated with autoimmune diseases including autoimmune thyroiditis and Sjogren's disease in this study. First degree relatives of $\mathrm{PBC}$ patients showed a higher rate of diagnosis of $\mathrm{PBC}$ than of those in the control group (6.2\% vs. ०\%) because familial clustering cases with four sibling PBC cases were included in this study [2o]. It might lead to a stronger association between family history of PBC in this study compared with other studies in which the prevalence of PBC in first degree relatives was reported to be $1.33 \%$ to $6.40 \%$ [21].

Many studies reported that recurrent UTI was a significant risk factor of PBC [5-7,15]. Escherichia coli, a major microorganism causing UTI, can be a strong inducer of pyruvate dehydrogenase complexes (PDC)-E2 specific AMA and liver pathology consistent with $\mathrm{PBC}$ in a mouse model [22]. However, ever or multiple UTIs were not associated with PBC in this study. Notably, the UTI rates in this study $(26.7 \%)$ were much lower than in other studies $(39 \%$ to $70 \%)$ [4,15]. It was not clear whether the lower rate of UTIIs reflected a really low incidence in Koreans or resulted from a recall bias; therefore, further validation is required.

Not only bacterial infection, but also xenobiotics may break immune tolerance by the mechanism of molecular mimicry. Octynoic acid, commonly used in artificial flavoring and cosmetics, was shown to induce AMA and PBC-like disease in murine models [23]. Moreover, certain xenobiotics such as nail polish [7] and hair dye [6] were reported to be associated with PBC. However, there were no clear associations between the use of xenobiotics including cosmetics, hair dye, perm agent, nail polish, and herbal drugs in this study.

Because PBC is a female predominant disease, many studies have explored the association between hormonal or reproductive factors and PBC. Some study reported gravidity [24], exogenous estrogen [7], and pruritus during pregnancy $[5,6]$ were associated with $\mathrm{PBC}$, and other reported oral contraceptives [5] were protective against PBC development. Most results on reproductive factors have not been reproduced in other studies. In this study, the proportion of females who had experienced receiving an induced abortion was strikingly high, and it was significantly more frequent in PBC patients than in the controls. The rate of induced abortions in Korea was reported to be up to $50 \%$ in 1980 s and 1990 s [25], whereas the use of oral contraceptives was reported to be $6 \%$ in 1984 [26] although it increased to $31.3 \%$ in 2007 in Korea [27]. Corpechot et al. [5] also reported that abortion was a risk factor of PBC. Moreover, induced abortion was reported to be a risk factor in autoimmune thyroiditis [28]. Fetal microchimerism, which occurs more frequently in surgical abortions than in spontaneous abortions, was assumed to be associated with the development of autoimmune diseases [29]. Therefore, the association between PBC and abortion should be studied further. Cultural differences in contraception methods might lead to the different association between PBC and reproductive factors among countries. Unexpectedly, the number as well as the experience of a full-term delivery was negatively associated with PBC. There have been few studies on the association between PBC and full-term delivery or gravity. One study showed that $\mathrm{PBC}$ patients reported significantly more pregnancies which is contradictory to our results. This association also needs to be identified further.

The limitations of this study include an interview-based questionnaire survey which has inherently a recall bias and a relatively small sample size. In addition, the $\mathrm{PBC}$ patients were enrolled from tertiary referral centers and might not be representative of $\mathrm{PBC}$ patients in general. Lastly, comorbidities and past illness can be underestimated because the PBC patients were interviewed by medical personnel, while the controls were interviewed by non-medical professional interviewers working for a commercial survey company, although we used the same questionnaires with detailed explanations and education to minimize the heterogeneity among the interviewers. Despite these limitations, this is the first study to our knowledge on the risk factors of PBC in Asian countries. Moreover, this is a multicenter study using face-to-face interviews for both the patients and the controls with comprehensive and detailed questionnaires that included reported risk factors on PBC.

In summary, we found that smoking, history of auto- 
immune disease, and artificial abortions were risk factors of $\mathrm{PBC}$, while alcohol drinking and full-term delivery were negatively associated with PBC in South Korea. Further studies to validate the results of this study and search for clues on the pathogenesis of PBC are warranted.

\section{KEY MESSAGE}

1. A family history of primary biliary cholangitis (PBC), accompanying autoimmune diseases, and smoking were significantly associated with $\mathrm{PBC}$ in this case-control study.

2. More induced abortions and less full-term deliveries were significantly associated with female PBC.

3. Mild to moderate alcohol intake was negatively associated with PBC.

\section{Conflict of interest}

No potential conflict of interest relevant to this article was reported.

\section{REFERENCES}

1. Poupon R. Primary biliary cirrhosis: a 2010 update. J Hepatol 2010;52:745-758.

2. Boonstra K, Beuers U, Ponsioen CY. Epidemiology of primary sclerosing cholangitis and primary biliary cirrhosis: a systematic review. J Hepatol 2012;56:1181-1188.

3. Bianchi I, Carbone M, Lleo A, Invernizzi P. Genetics and epigenetics of primary biliary cirrhosis. Semin Liver Dis 2014;34:255-264.

4. Lammert C, Nguyen DL, Juran BD, et al. Questionnaire based assessment of risk factors for primary biliary cirrhosis. Dig Liver Dis 2013;45:589-594.

5. Corpechot C, Chretien Y, Chazouilleres O, Poupon R. Demographic, lifestyle, medical and familial factors associated with primary biliary cirrhosis. J Hepatol 2010;53:162169.

6. Prince MI, Ducker SJ, James OF. Case-control studies of risk factors for primary biliary cirrhosis in two United Kingdom populations. Gut 2010;59:508-512.

7. Gershwin ME, Selmi C, Worman HJ, et al. Risk factors and comorbidities in primary biliary cirrhosis: a con- trolled interview-based study of 1032 patients. Hepatology 2005;42:1194-1202.

8. Kim KA, Ki M, Choi HY, Kim BH, Jang ES, Jeong SH. Population-based epidemiology of primary biliary cirrhosis in South Korea. Aliment Pharmacol Ther 2016;43:154162.

9. Selmi C, Mayo MJ, Bach N, et al. Primary biliary cirrhosis in monozygotic and dizygotic twins: genetics, epigenetics, and environment. Gastroenterology 2004;127:485-492.

10. Mantaka A, Koulentaki M, Chlouverakis G, et al. Primary biliary cirrhosis in a genetically homogeneous population: disease associations and familial occurrence rates. BMC Gastroenterol 2012;12:110.

11. Invernizzi P. Human leukocyte antigen in primary biliary cirrhosis: an old story now reviving. Hepatology 2011;54:714-723.

12. Corpechot C, Gaouar F, Chretien Y, Johanet C, Chazouilleres O, Poupon R. Smoking as an independent risk factor of liver fibrosis in primary biliary cirrhosis. J Hepatol 2012;56:218-224.

13. Ng M, Freeman MK, Fleming TD, et al. Smoking prevalence and cigarette consumption in 187 countries, 19802012. JAMA 2014;311:183-192.

14. Howel D, Fischbacher CM, Bhopal RS, Gray J, Metcalf JV, James OF. An exploratory population-based case-control study of primary biliary cirrhosis. Hepatology 2000;31:10551060.

15. Parikh-Patel A, Gold EB, Worman H, Krivy KE, Gershwin ME. Risk factors for primary biliary cirrhosis in a cohort of patients from the united states. Hepatology 2001;33:1621.

16. Boonstra K, Kunst AE, Stadhouders PH, et al. Rising incidence and prevalence of primary biliary cirrhosis: a large population-based study. Liver Int 2014;34:e31-e38.

17. Zein CO, Unalp A, Colvin R, Liu YC, McCullough AJ; Nonalcoholic Steatohepatitis Clinical Research Network. Smoking and severity of hepatic fibrosis in nonalcoholic fatty liver disease. J Hepatol 2011;54:753-759.

18. Anaya JM, Restrepo-Jimenez P, Ramirez-Santana C. The autoimmune ecology: an update. Curr Opin Rheumatol 2018;30:350-360.

19. Floreani A, Restrepo-Jimenez P, Secchi MF, et al. Etiopathogenesis of autoimmune hepatitis. J Autoimmun 2018;95:133-143.

20. Shin S, Moh IH, Woo YS, et al. Evidence from a familial case suggests maternal inheritance of primary biliary 
cholangitis. World J Gastroenterol 2017;23:7191-7197.

21. Mells GF. Primary biliary cirrhosis: family, genes, and bugs. Clin Liver Dis (Hoboken) 2014;3:69-73.

22. Wang JJ, Yang GX, Zhang WC, et al. Escherichia coli infection induces autoimmune cholangitis and anti-mitochondrial antibodies in non-obese diabetic (NOD).B6 (Idd10/Idd18) mice. Clin Exp Immunol 2014;175:192-201.

23. Wakabayashi K, Lian ZX, Leung PS, et al. Loss of tolerance in $\mathrm{C}_{57} \mathrm{BL} / 6$ mice to the autoantigen E2 subunit of pyruvate dehydrogenase by a xenobiotic with ensuing biliary ductular disease. Hepatology 2008;48:531-540.

24. Parikh-Patel A, Gold E, Utts J, Gershwin ME. The association between gravidity and primary biliary cirrhosis. Ann Epidemiol 2002;12:264-272.
25. Jeon HS, Seo HG. Abortion in Korea since 1945. Uisahak 2003;12:129-143.

26. Park KH. Current status of contraception. Korean J Fertil Steril 1986;13:95-100.

27. Lee DY, Koo YA, Yoon BK, Choi D. Reproductive health characteristics of urban South Korean women. Gynecol Obstet Invest 2010;70:154-159.

28. Carle A, Pedersen IB, Knudsen N, et al. Development of autoimmune overt hypothyroidism is highly associated with live births and induced abortions but only in premenopausal women. J Clin Endocrinol Metab 2014;99:2241-2249.

29. Miech RP. The role of fetal microchimerism in autoimmune disease. Int J Clin Exp Med 2010;3:164-168. 
Supplementary Material . Questionnaire survey form (in Korean). 